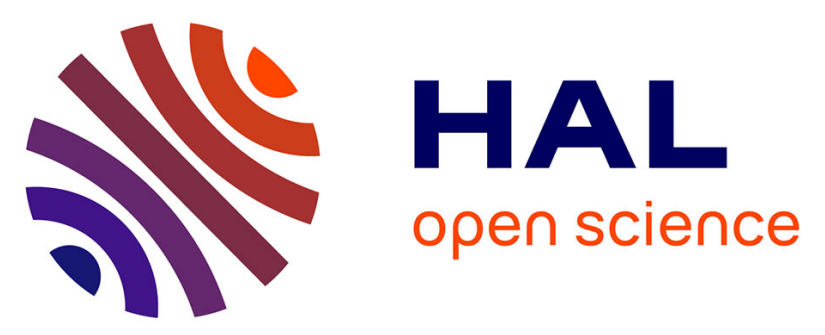

\title{
A displacement-pressure finite element formulation for analyzing the sound transmission in ducted shear flows with finite poroelastic lining
}

Benoit Nennig, Mabrouk Ben Tahar, Emmanuel Perrey-Debain

\section{- To cite this version:}

Benoit Nennig, Mabrouk Ben Tahar, Emmanuel Perrey-Debain. A displacement-pressure finite element formulation for analyzing the sound transmission in ducted shear flows with finite poroelastic lining. Journal of the Acoustical Society of America, 2011, 130 (1), pp.42-51. 10.1121/1.3598451 . hal-00694763

\section{HAL Id: hal-00694763 \\ https://hal.science/hal-00694763}

Submitted on 6 May 2012

HAL is a multi-disciplinary open access archive for the deposit and dissemination of scientific research documents, whether they are published or not. The documents may come from teaching and research institutions in France or abroad, or from public or private research centers.
L'archive ouverte pluridisciplinaire HAL, est destinée au dépôt et à la diffusion de documents scientifiques de niveau recherche, publiés ou non, émanant des établissements d'enseignement et de recherche français ou étrangers, des laboratoires publics ou privés. 


\title{
A displacement-pressure finite element formulation for analyzing the sound transmission in ducted shear flows with finite poroelastic lining.
}

\author{
Benoit Nennig, a) Mabrouk Ben Tahar, and Emmanuel Perrey-Debain \\ Université de Technologie de Compiègne, Laboratoire Roberval UMR 6253, BP 20529, 60205 Compiègne cedex, \\ France.
}

(Dated: May 6, 2012)

\begin{abstract}
In the present work, the propagation of sound in a lined duct containing sheared mean flow is studied. Walls of the duct are acoustically treated with absorbent poroelastic foams. The propagation of elasto-acoustic waves in the liner is described by the Biot's model. In the fluid domain, the propagation of sound in a sheared mean flow is governed by the Galbrun's equation. The problem is solved using a mixed displacement-pressure finite element formulation in both domains. A 3D implementation of the model has been performed, and is illustrated on axisymmetric examples. Convergence and accuracy of the numerical model are shown for the particular case of the modal propagation in a infinite duct containing an uniform flow. Practical examples concerning the sound attenuation through dissipative silencers are discussed. In particular, effects of the refraction effects in the shear layer as well as the mounting conditions of the foam on the Transmission Loss are shown. The presence of a perforate screen at the air-porous interface is also considered and included in the model.
\end{abstract}

PACS numbers: 43.20.Mv, 43.50.Gf, 43.28.Py

\section{INTRODUCTION}

Over the years, considerable effort has been directed toward the development of finite element (FE) models for predicting the propagation of sound waves in gas flow in the presence of acoustically treated walls ${ }^{1-4}$. These treatments are generally multi-layered and composed of perforated plates backed by honey comb or backed with a porous blanket. Porous materials are generally used to broaden the absorption frequency range of the liner. The practical applications of such work range from noise transmission in vehicle exhaust systems, through ventilation and air conditioning ducts, to the prediction of more complex three-dimensional fields aircraft engine-duct systems.

In the early work dealing with absorption and FE, it was assumed that the liner was locally reacting, thus eliminating the need to discretize the absorbing material explicitly. Later, to illustrate the effects of a finite size bulk reacting lining, Craggs $^{5}$ derived a finite element model for motionless porous materials. This model is well adapted for rigid materials. The solid frame can also be assumed to be infinitely soft and the so-called limp model $^{6,7}$ must be used; the stiffness of the solid phase is neglected but its inertial effects are conserved. In both cases, only one compressional wave is allowed to propagate in the material; these are known as fluid equivalent models and can be characterized by a complex wave number and a complex characteristic impedance ${ }^{6,8,9}$. For a wide class of aborbent materials such as polymer foams, the solid structure has a finite stiffness. In this case the Biot's theory ${ }^{10,11}$ describing the propagation of elastic and pressure waves in poroelastic material must be

\footnotetext{
a)Electronic address: benoit.nennig@utc.fr
}

used. The numerical solution of Biot's equations using the finite element method with the $h$ or $p$ version have been extensively discussed in the literature and various FE formulations involving different variables have been proposed $^{12-15}$. In this regard, the Atalla et al.'s mixed $\left(\mathbf{u}, p_{p}\right)$ formulation has several advantages of reducing the number of degrees of freedom as well as easing the transmission conditions at the air-porous interface ${ }^{13,16}$. It is remarkable that most of the studies related to sound attenuation through dissipative silencers are carried out using simplified fluid equivalent models and that no attempt was made to quantify the effects of the finite stiffness of the absorbent material. The first paper in this matter seems to be that of Kang ${ }^{17}$ for circular ducts. The authors developed an axisymmetric finite element formulation for poroelastic liner and showed that mounting conditions can have great impact on the sound attenuation.

All references cited above do not consider the presence of a gas flow in the fluid domain. Ignoring the convection and refraction effects due to the mean flow can lead to erroneous results especially for relatively high speed flow. Indeed, the presence of a flow in the airway is known to have a non negligible effect on the upstream and downstream propagation. This has been investigated in a modal context for infinite lined circular duct ${ }^{18}$, slowly varying porous liner ${ }^{19}$ or using $\mathrm{FE}$ mode solvers for ducts with arbitrary cross section ${ }^{1}$. For lining of finite dimension, modal matching methods can sometimes be used for a certain class of problems ${ }^{18,20}$. In most work, the mean flow is considered uniform in the duct. This simplification is made to ease the numerical treatment of the wave equation, but it has the drawback to neglect refraction effects due to the rotational flow in the boundary layer which is inevitably present in the vicinity of the walls $^{21}$. In fact very little research have dealt with modeling the wave propagation inside the absorbent material 
exposed to a grazing shear flow and the work is usually restricted to the modal analysis of infinite duct by solving the Pridmore-Brown equation ${ }^{22,23}$.

Because of these limitations, we think there is a need to develop more advanced numerical techniques based on the finite element methodology in order to incorporate these effects. For a general rotational mean flow, two equivalent physical models are proposed in the literature: the Linearized Euler's Equations (LEE) and the Galbrun's equation ${ }^{24,25}$. The numerical solution of LEE can be carried out either in the frequency or in the time domain. For this latter, however, there remain some stability issues regarding propagation through shear layers and the correct implementation of time-domain impedance boundary conditions. Some interesting discussions can be found in a recent review of Astley ${ }^{26}$ and in references therein. Galbrun's equation offers an interesting alternative to LEE. The equation describes exactly the same physical phenomenon as the LEE but is obtained using a different linearization process involving the displacement perturbation, instead of the acoustic velocity, as primitive variable. All details of the formulation as well as its FE discretization can be found in Refs. 24, 25, 27, 28 and some aspects will be discussed and reminded later.

In the context of poroelastic liners exposed to a grazing flow, Galbrun's equation offers several advantages: (i) it allows a direct treatment of the coupling condition at the interface as the continuity of normal acoustic displacement appears explicitly in the boundary terms ${ }^{25,29}$, (ii) it can be written as an exact conservation law for homentropic rotational flow and a definition of the acoustic intensity is available ${ }^{25,27}$, (iii) efficient and stable FE formulations in the frequency domain are available ${ }^{28}$. All this makes the method a candidate of choice for dealing with the presence of poroelastic materials which are defined via frequency dependent Biot's parameters.

Based on previous studies, the aim of the present work is to extend and propose a FE formulation for analyzing the sound transmission in ducted shear flows with finite poro-elastic lining. The propagation of waves in the liner is described via the Biot's model whereas the sound propagation in the fluid domain is governed by the Galbrun's equation. The problem is solved for a general 3D configuration using a mixed displacement-pressure finite element formulation in both domains allowing an efficient treatment of the coupling conditions at the air-porous interface. Details of physical models and their FE formulations are presented in Section 2 under the assumptions there is no induced flow in the liner. Convergence and accuracy of the numerical model are shown in Section 3 for the particular case of the modal propagation in a infinite duct containing an uniform flow. To illustrate the method, we compute the Transmission Loss for typical dissipative silencers encountered in the automotive industry and this is presented in Section 4. We show that the existence of multiple wave types in the porous material can have significative consequences on the sound attenuation and that the liner is very sensitive to the boundary conditions that exist at its surface. The presence of a perforate screen at the air-porous interface is also considered and included in the FE model. Compu- tational aspects of the method are discussed at the end of the paper.

\section{PROBLEM FORMULATION}

\section{A. Problem statement}

The configuration considered here consists of a dissipative silencer comprising a expansion chamber of length $L$ filled with a sound-absorbing poroelastic material. In the airway, a sheared mean flow with speed $\mathbf{v}_{0}$ is present. In this work, we restrict the analysis to parallel shear flow where the density $\rho_{0}$, the sound wave speed $c_{0}$ and the mean pressure $p_{0}$ are taken constant ${ }^{30}$ (Eq. (1.12)). Furthermore, we consider problems with axial symmetry, so we can define the local Mach number as a function of the radial coordinate only: $M(r)=v_{0}(r) / c_{0}$. The inlet and outlet rigid wall pipes are identical, each having a circular cross section with radius $r_{1}$. This simple configuration have been chosen for a sake of illustration, but the numerical scheme presented in this paper can be used for solving general 3D problems with non potential flow as in Ref. 31.

For a brief nomenclature, we call $\Omega_{a}$ and $\Gamma_{a}$, the airflow flow domain $\left(r \leq r_{1}\right)$ and its boundary. Similarly, we call $\Omega_{p}$ and $\Gamma_{p}$ the poroelastic domain and its boundary. The acoustic field is generated by an incident field stemming from the left. On both ends $\Gamma_{i}$ and $\Gamma_{o}$, appropriate modal conditions must be used to ensure that the reflected and transmitted fields are radiating away from the dissipative silencer. The mathematical formulation for this will be stated later. Finally we call $\Gamma$ the air-porous interface and $\Gamma_{w}$ denotes the rigid walls.

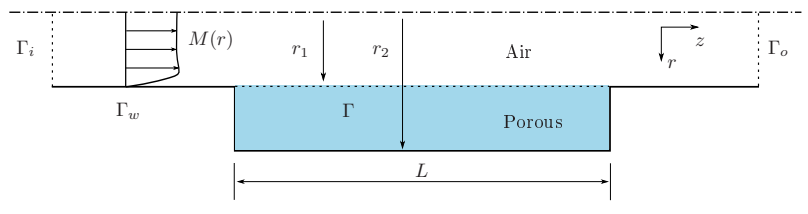

FIG. 1. Geometry of the silencer benchmark.

\section{B. Galbrun's equation}

Galbrun's equation derives from general fluid mechanic conservation equations, whereby the linearization process is carried out with a lagrangian perturbation of the displacement ${ }^{24,25,27,28}$. Under the previous assumptions and without the presence of acoustic sources, the Galbrun's equation for the displacement perturbation $\mathbf{w}$ and the acoustic pressure $p$ can be written in the frequency domain $\left(\mathrm{e}^{-\mathrm{i} \omega t}\right)$ as follows :

$$
\begin{aligned}
& \rho_{0} \frac{\mathrm{d}_{0}^{2} \mathbf{w}}{\mathrm{d} t^{2}}+\nabla p=0, \\
& p=-\rho_{0} c_{0}^{2} \nabla \cdot \mathbf{w}
\end{aligned}
$$


where $\frac{\mathrm{d}_{0}}{\mathrm{~d} t} \equiv-\mathrm{i} \omega+\mathbf{v}_{0} \cdot \nabla$ is the material derivative along the mean flow.

One may observe that it is possible to express (1) with the displacement perturbation only. However solving the Galbrun's equation in this form with conventional nodal finite element is known to give rise to corrupted results. Thus, to overcome the so-called locking effects in the FE formulation two ways have been investigated in the past (i) the regularization technique ${ }^{32}$, which add a special treatment for the curl of the displacement. (ii) The pressure is kept as an independent variable as in Ref. 28: this is the displacement-pressure mixed formulation of Galbrun's equation. This approach will be used in the following. The interested reader may consults Ref. 28, 32 and the references therein.

After integration by parts, the associated weak formulation to the mixed form is

$$
\begin{gathered}
-\int_{\Omega_{a}} \rho_{0} \frac{\overline{\mathrm{d}}_{0} \mathbf{w}^{*}}{\mathrm{~d} t} \cdot \frac{\mathrm{d}_{0} \mathbf{w}}{\mathrm{d} t} \mathrm{~d} \Omega+\int_{\Omega_{a}} \mathbf{w} \cdot \nabla p^{*} \mathrm{~d} \Omega \\
\quad+\int_{\Omega_{a}} \nabla p \cdot \mathbf{w}^{*} \mathrm{~d} \Omega-\int_{\Omega_{a}} \frac{p^{*} p}{\rho_{0} c_{0}^{2}} \mathrm{~d} \Omega \\
+\int_{\Gamma_{a}} \rho_{0}\left(\mathbf{v}_{0} \cdot \mathbf{n}^{\prime}\right) \frac{\mathrm{d}_{0} \mathbf{w}}{\mathrm{d} t} \cdot \mathbf{w}^{*} \mathrm{~d} \Gamma-\int_{\Gamma_{a}} p^{*} \mathbf{w} \cdot \mathbf{n}^{\prime} \mathrm{d} \Gamma=0,
\end{gathered}
$$

for all the test functions $p^{*}, \mathbf{w}^{*}$. Here, $\mathbf{n}^{\prime}$ is the outward normal unit vector and the bar symbol on the material derivative means that we take the complex conjugate, i.e. $\frac{\overline{\mathrm{d}}_{0}}{\mathrm{~d} t} \equiv \mathrm{i} \omega+\mathbf{v}_{0} \cdot \nabla$.

\section{Biot's model}

The wave propagation involving respectively the fluid and solid phases displacement $\mathbf{U}$ and $\mathbf{u}$ in the poroelastic medium is described by the Biot-Allard model which is well documented in the reference textbook ${ }^{11}$. For the numerical implementation, we use the Atalla's mixed $\left(\mathbf{u}, p_{p}\right)$ formulation $^{13,16}$. This has the advantage of reducing the number of degrees of freedom as well as easing the transmission conditions at the air-porous interface. This reduced formulation reads

$$
\begin{aligned}
\nabla \cdot \boldsymbol{\sigma}^{s}(\mathbf{u})+\omega^{2} \rho \mathbf{u}+\gamma \nabla p_{p} & =0 \\
\Delta p_{p}+\omega^{2} \frac{\rho_{22}}{R} p_{p}-\omega^{2} \frac{\rho_{22}}{\phi^{2}} \gamma \nabla \cdot \mathbf{u} & =0 .
\end{aligned}
$$

Here, $p_{p}$ is the pore pressure, $\phi$ is porosity of the porous material, $\gamma=\phi\left(\frac{\rho_{12}}{\rho_{22}}-\frac{Q}{R}\right)$ and $\rho=\rho_{11}-\frac{\rho_{12}^{2}}{\rho_{22}}$. The effective density coefficient $\rho_{11}, \rho_{22}$, respectively for the solid phase and the fluid phase, and the coupling density coefficient $\rho_{12}$, are complex and the imaginary part takes into account viscous damping. The in vacuo stress tensor $\boldsymbol{\sigma}^{s}$ reads

$$
\boldsymbol{\sigma}^{s}(\mathbf{u})=\mathbf{I}\left(K_{b}-\frac{2}{3} N\right) \nabla \cdot \mathbf{u}+2 N \varepsilon^{s}(\mathbf{u})
$$

with $\varepsilon^{s}$ the in vacuo strain tensor. Here, $K_{b}$ is the complex dynamic bulk modulus of the frame and takes into account the thermal dissipation. In the same way, the shear modulus $N$ includes the structural damping. Lastly, $R$ is the effective bulk modulus of the fluid phase and $Q$ indicates the coupling of the two phases volumic dilatation. All these coefficients are related to the poroelastic structural parameters (see Table III) by the Johnson-Champoux-Allard model and can be found in Ref. 11.

Given tests functions $p_{p}^{*}, \mathbf{u}^{*}$, the associated weak formulation is ${ }^{13}$

$$
\begin{aligned}
& \int_{\Omega_{p}} \boldsymbol{\sigma}^{s}(\mathbf{u}): \varepsilon^{s}\left(\mathbf{u}^{*}\right) \mathrm{d} \Omega-\omega^{2} \int_{\Omega_{p}} \rho \mathbf{u} \cdot \mathbf{u}^{*} \mathrm{~d} \Omega \\
& +\int_{\Omega_{p}}\left[\frac{\phi^{2}}{\omega^{2} \rho_{22}} \nabla p_{p} \cdot \nabla p_{p}^{*}-\frac{\phi^{2}}{R} p_{p} p_{p}^{*}\right] \mathrm{d} \Omega \\
& -\int_{\Omega_{p}}\left(\gamma+\phi^{\prime}\right)\left(\nabla p_{p}^{*} \cdot \mathbf{u}+\nabla p_{p} \cdot \mathbf{u}^{*}\right) \mathrm{d} \Omega \\
& -\int_{\Omega_{p}} \phi^{\prime}\left(p_{p}^{*} \nabla \cdot \mathbf{u}+p_{p} \nabla \cdot \mathbf{u}^{*}\right) \mathrm{d} \Omega \\
& -\underbrace{\int_{\Gamma_{p}} \boldsymbol{\sigma}^{t} \mathbf{n} \cdot \mathbf{u}^{*} \mathrm{~d} \Gamma}_{I_{\sigma}}-\int_{\Gamma_{p}} \phi(\mathbf{U}-\mathbf{u}) \cdot \mathbf{n} p_{p}^{*} \mathrm{~d} \Gamma=0 .
\end{aligned}
$$

We put $\phi^{\prime}=\phi(1+Q / R)$ and $\mathbf{n}$ denotes the outward normal unit vector to the poroelastic domain. Note that the presence of the boundary integral involves the fluid normal displacement $\mathbf{U} \cdot \mathbf{n}$ and the total stress tensor $\boldsymbol{\sigma}^{t}$. These new physical quantities do not need to be stated explicitly here as they will be replaced after applying boundary conditions.

\section{Coupling conditions and global formulation}

Coupling conditions between the acoustic domain and the poroelastic material are summarized by Debergue et $a l .{ }^{33}$ :

$$
\begin{aligned}
& \boldsymbol{\sigma}^{t} \mathbf{n}=-p \mathbf{n} \\
& p_{p}=p \\
& \mathbf{w} \cdot \mathbf{n}=\phi(\mathbf{U}-\mathbf{u}) \cdot \mathbf{n}+\mathbf{u} \cdot \mathbf{n} .
\end{aligned}
$$

The first condition is the standard continuity requirement of the normal stress at the interface. The second condition ensures the continuity of the pressure between the acoustic domain and the pores. The last condition ensures the continuity of the displacement at the interface. Though these conditions have been specified in no-flow case $^{33}$, they still hold when a flow is present in the airway $^{25,34}$.

On the hard wall of the expansion chamber $\Gamma_{w}$, two scenarios are investigated (i) the porous foam is considered clamped, that is the solid phase displacement vector is zero at the wall and (ii) the foam is sliding and only the normal component of the displacement is set to zero. In both cases, the boundary terms in (5) vanish on $\Gamma_{w}$ since $\mathbf{u}^{*}$ is set to zero.

The global formulation is obtained by summing (2) and (5). Because the expressions are quite lengthy, we 
shall detail only the boundary terms on the air-porous interface $\Gamma$. First, since there is no flow in the liner, $\mathbf{v}_{0}$ and $\mathbf{n}^{\prime}$ are orthogonal and the first boundary term in (2) (its restriction on $\Gamma$ ) vanishes. Both conditions (6a) and (6c) are directly substituted in the boundary integral over $\Gamma$. For the condition of continuity of pressure, we introduce an additional functional

$$
\int_{\Gamma} \mathbf{w}^{*} \cdot \mathbf{n}\left(p-p_{p}\right) \mathrm{d} \Gamma=0,
$$

where $\mathbf{w}^{*}$ can be interpreted as a Lagrange multiplier. Now, (6b) is added thanks to (7) and we can switch $p$ and $p_{p}$ in the boundary integral term $I_{\sigma}$ in (5). These two manipulations give a symmetric formulation of the coupling integrals. We finally arrive at the weak form for the coupling on $\Gamma$ :

$$
\begin{array}{r}
\int_{\Gamma} p_{p} \mathbf{u}^{*} \cdot \mathbf{n} \mathrm{d} \Gamma+\int_{\Gamma} p_{p}^{*} \mathbf{u} \cdot \mathbf{n} \mathrm{d} \Gamma+\int_{\Gamma} p \mathbf{w}^{*} \cdot \mathbf{n} \mathrm{d} \Gamma+\int_{\Gamma} p^{*} \mathbf{w} \cdot \mathbf{n} \mathrm{d} \Gamma \\
-\int_{\Gamma} p_{p}^{*} \mathbf{w} \cdot \mathbf{n} \mathrm{d} \Gamma-\int_{\Gamma} p_{p} \mathbf{w}^{*} \cdot \mathbf{n} \mathrm{d} \Gamma=0
\end{array}
$$

The first line of (8) corresponds to a displacementpressure coupling within each separate domain whereas the second line represents the displacement-pressure coupling between the two domains. It can be noted the two displacement-pressure weak formulations (5) and (2) make easier the coupling between both domain and this is due to the continuity of the lagrangian perturbation of the displacement. In particular, this allows easy treatment of plug flows. In this latter case, the use of the LEE would have been more difficult as the Myers boundary condition ${ }^{35}$ is not taken into account naturally.

The combination of (5), (2) and (8) yields the coupled fluid-poroelastic finite element system which can be written in the compact form

$$
\left(\begin{array}{cc}
\mathbf{G} & \mathbf{C} \\
\mathbf{C}^{\mathrm{t}} & \mathbf{B}
\end{array}\right)\left(\begin{array}{c}
p \\
\mathbf{w} \\
\hline p_{p} \\
\mathbf{u}
\end{array}\right)=\left(\begin{array}{l}
\mathbf{F}_{\mathbf{G}} \\
\mathbf{F}_{\mathbf{B}}
\end{array}\right)
$$

One can note that the discretization of the Galbrun's equation in its mixed form leads to the complex hermitian matrix block G. However the displacement-pressure formulation of Biot's equations gives a complex symmetric matrix $\mathbf{B}$. The right hand sides vectors $\mathbf{F}_{\mathbf{G}}$ and $\mathbf{F}_{\mathbf{B}}$ arise from the boundary conditions. In this study, we have necessarily $\mathbf{F}_{\mathbf{B}}=\mathbf{0}$ and $\mathbf{F}_{\mathbf{G}}$ stems from the pressuredisplacement condition at the inlet and outlet planes.

\section{VALIDATION AND ASSESSMENT OF THE FE MODEL}

In order to validate and assess the FE model, we shall consider the case of an infinite cylindrical silencer.On the outer radius $r_{2}$, the foam is clamped and there is an uniform mean flow with Mach number $M$ in the airway. In this scenario, the problem is separable in cylindrical coordinates $(r, \theta, z)$ and analytical modal solutions can be found. These solutions all have the $\mathrm{e}^{\mathrm{i}(\beta z-\omega t)}$ dependence and finding these modes requires the computation of the axial wavenumbers $\beta$. In appendix $A$, the method for finding these modes is presented very briefly for the case of purely radial modes only. We refer the reader to Ref. 20 for more details.

Now, for a chosen mode, the analytical solution for both the pressure and the displacement vector is imposed directly in the system (9) at nodes corresponding to the inlet and outlet sections $\Gamma_{i}$ and $\Gamma_{o}$. A convergence rate study has been carried out for three different discretization schemes listed in Table I. Biot's equations are discretized with standard isoparametric tetrahedral elements called T4 and T10 using respectively a linear and a quadratic lagrangian polynomial interpolation basis for the pressure and for the solid displacement field. To ensure the convergence, the mixed formulation (2) is discretized with an appropriate choice for the interpolation function basis satisfying the inf-sup condition ${ }^{36}$. For this purpose, the so-called T5-4c and T10-4c mixed elements are used ${ }^{28,36,37}$. In the T5-4c element (also called "MINI") the pressure interpolation is linear while the displacement field is enriched with a bubble function. In the T10-4c, the pressure is linear whereas the displacement is interpolated with quadratic basis functions.

For a given physical quantity $X$, we define the relative error estimator $\mathcal{E}_{2}$ in terms of the energy-norm as

$$
\mathcal{E}_{2}(X)=100 \frac{\left\|X^{\text {fem }}-X^{\text {ana }}\right\|_{L^{2}(S)}}{\left\|X^{\text {ana }}\right\|_{L^{2}(S)}}
$$

where $S$ a surface of reference lying on the $\theta=0$ plane and corresponding either to $\Omega_{a}$ or $\Omega_{p}$.

TABLE I. Three discretisation schemes.

\begin{tabular}{ccc}
\hline \hline Scheme & $\Omega_{a}$ & $\Omega_{p}$ \\
\hline LL & T5-4c & T4 \\
LQ & T5-4c & T10 \\
QQ & T10-4 & T10 \\
\hline \hline
\end{tabular}

A comparison between the analytical solution and the FE solution computed with the LQ scheme is shown in Fig. 2. The dimensions are $r_{1}=0.06 \mathrm{~m}, r_{2}=0.1 \mathrm{~m}$ with a the total length of $L=0.6 \mathrm{~m}$. In all cases the frequency is fixed at $600 \mathrm{~Hz}$. For all physical quantities we can note a good agreement between the computed values and the modal solution. This is confirmed by the relative error $\mathcal{E}_{2}$ given in Table II. In this example, approximately eight elements were used in the radial direction. In this regard, the FE mesh is shown on the reference surface in order to give an idea of the discretization level. Note that the pressure is continuous across the interface whereas the normal displacement presents a jump due to the separation of the total displacement field into the fluid and solid phase.

A convergence rate analysis has been performed for two configurations (i) a no-flow case with mode $\beta=$ $17.135+4.7643 \mathrm{i}$ and (ii) an upstream propagation case 

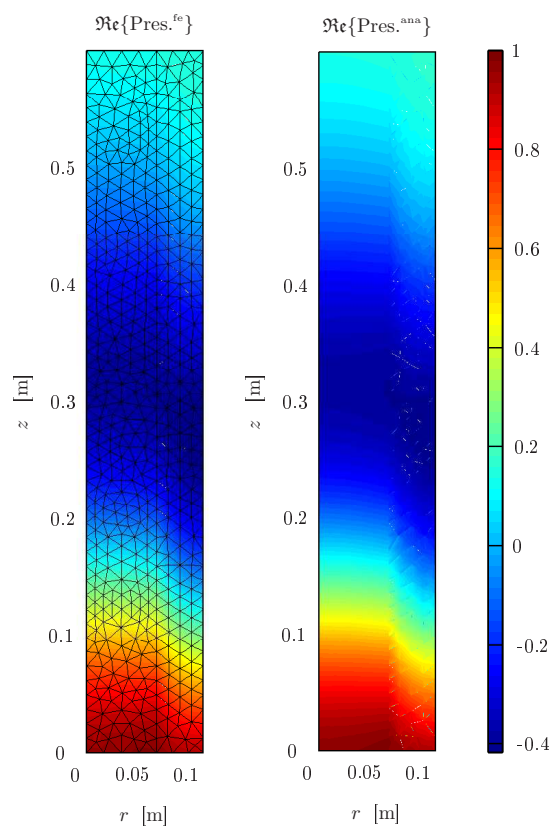

(a)
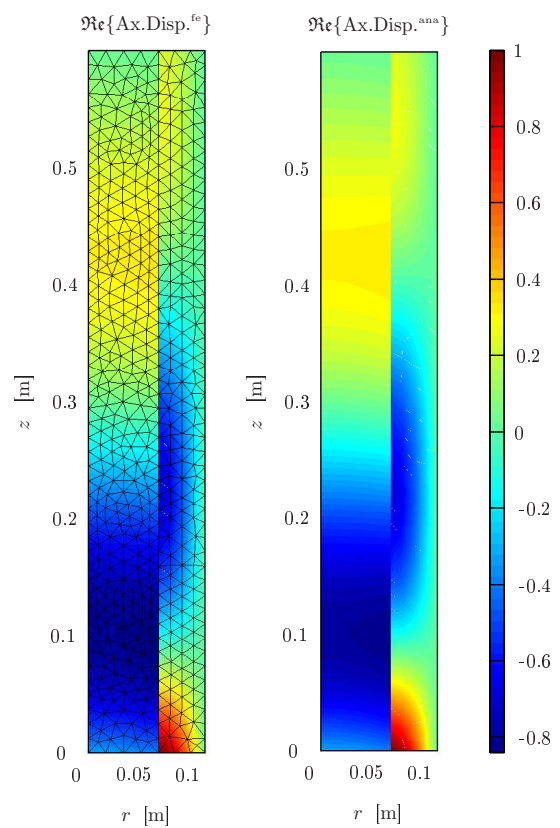

(b)
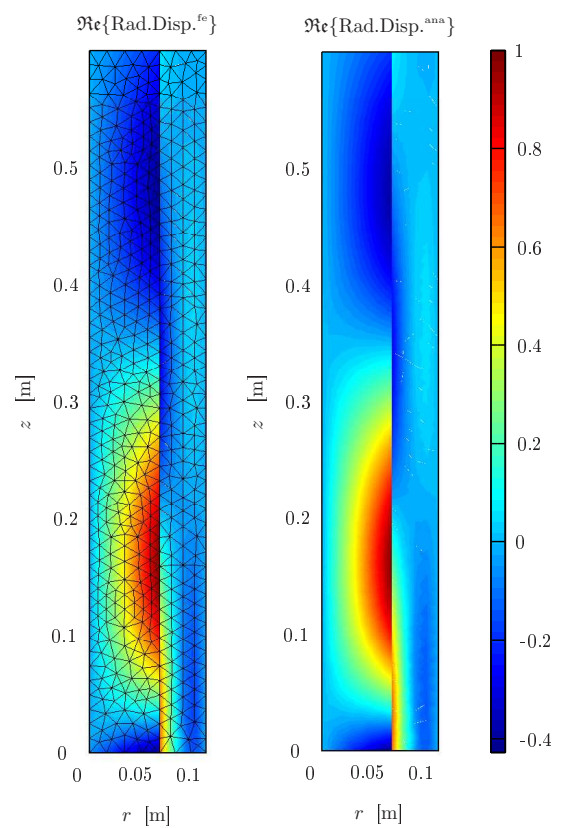

(c)

FIG. 2. Numerical results (fe) computed with the LQ scheme and analytical solutions (ana) for the real part of the pressure, the axial and the radial displacement (quantities are scaled for the sake of clarity). The displacement 'Disp.' (resp. the pressure 'Pres.') signifies the fluid displacement perturbation in the airway w (resp. pressure $p$ ) for $r \leq r_{1}$, and the solid phase displacement $\mathbf{u}$ for $r_{1}<r \leq r_{2}$ (resp. pore pressure $p_{p}$ ). Computed with $\beta=9.7112+2.8826 \mathrm{i}, M=0.3$ and $f=600 \mathrm{~Hz}$.

TABLE II. Error $\mathcal{E}_{2}$ for $M=0.3, f=600 \mathrm{~Hz}, \beta=9.7112+$ 2.8826 i computed with the LQ scheme. Displacement 'Disp.' (resp. 'Pressure') signifies the fluid displacement perturbation in the airway $\mathbf{w}$ (resp. the pressure $p$ ), and the solid phase displacement $\mathbf{u}$ in the poroelastic material (resp. the pore pressure $\left.p_{p}\right)$.

\begin{tabular}{cccc}
\hline \hline Error $\mathcal{E}_{2}(\%)$ & Pressure & Radial Disp. & Axial Disp. \\
\hline Poroelastic mat. & 0.586 & 0.785 & 0.525 \\
Airway & 1.372 & 2.718 & 0.847 \\
\hline \hline
\end{tabular}

with $M=-0.2$ and mode $\beta=20.2572+7.1227$ i. Results for the pressure in both domains are conveniently plotted in Fig. 3. Errors in the displacement have been found to be higher than for the pressure but the convergence curves show similar trends. Clearly, best results have been obtained when using either LQ or QQ schemes. This indicates that quadratic interpolation for the poroelastic material discretization must be used in order to reduce computational costs and that errors of about one percent for the pressure in the fluid domain can be expected at a reasonable cost. Thereafter, the LQ scheme is used in all calculations.

Note that validations were also performed on silencer configurations with uniform mean flow and clamped boundary conditions. Comparisons with the recent mode matching technique proposed in Ref. 20 show a good agreement (see Figs. 8-12 therein).

\section{RESULTS}

\section{A. Test case configuration}

As a test case we consider the geometry already studied by Kirby ${ }^{18}$ so we take $r_{1}=37 \mathrm{~mm}, r_{2}=76.2 \mathrm{~mm}$ and $L=315 \mathrm{~mm}$. To illustrate the method we consider two foams which are standard polymer foams presenting a strong coupling between both fluid and solid phases: a 'soft' one called FM4 and the XFM foam. The material properties can be found in Refs. 16, 33 and are reminded in Table III for completeness. Here the diameter of the acoustic duct is relatively small and the first cut-off frequency is well above the frequency range of interest. Only the fundamental mode is allowed to propagate and this simplifies the radiating conditions at both ends of the duct. At the exit plane, the non-reflective condition is assured by the modal impedance of the first mode ${ }^{28}$ of the rigid duct. In an uniform flow, this mode is given analytically in terms of Bessel functions. In a shear flow, the mode is computed by solving the Pridmore-Brown equation using a shooting method as in ${ }^{28,38}$. At the inlet plane, the pressure-displacement field is expressed as a sum of an incident and a reflected mode and the global matrix is rearranged so that the incident term appears on the right-hand side $\mathbf{F}_{\mathbf{G}}$ of the $\mathrm{FE}$ system. Note that the same procedure can be followed in a multimodal context as shown in Ref. 39.

On Fig. 4, the test case mesh is shown. Since the problem possesses axial symmetry, only one quarter of 
the duct is meshed and normal displacement field are set to zero on the lateral sides (corresponding to $\theta=0$ and $\theta=\pi / 2)$.

In the present work, we wish to identify the effects of the poroelastic material as well as the presence of the flow in the airway on the Transmission Loss (TL) of the dissipative silencer. By calling $\mathcal{P}_{i}$ and $\mathcal{P}_{t}$ the incident and transmitted power, the TL is defined by

$$
\mathrm{TL}=-10 \log \left(\frac{\mathcal{P}_{t}}{\mathcal{P}_{i}}\right) .
$$

In the presence of a shear flow in the airway, the calculation for the transmitted and incident acoustic powers relies on the numerical evaluation of the intensity vector ${ }^{40}$

$$
\mathcal{I}=\rho_{0}\left(\frac{\partial \mathbf{w}}{\partial t} \cdot \frac{\mathrm{d}_{0} \mathbf{w}}{\mathrm{d} t}\right) \mathbf{v}_{\mathbf{0}}+p \frac{\partial \mathbf{w}}{\partial t}
$$

The powers are obtained after integration of the normal component (along the $z$-axis) of the intensity over the inlet and the outlet planes.

\section{B. Mounting conditions and skeleton influence}

It is known that the manner in which lining materials are fitted into the duct can have a great impact on the sound attenuation level ${ }^{17}$, especially with poroelastic materials presenting a strong skeleton influence. This effect has been experimentally observed and discussed in a standing wave tube ${ }^{41}$ and some criteria have been proposed to quantify the frame stiffness influence ${ }^{7,41}$.

To illustrate this, the clamped and sliding mounting conditions are compared and results on the TL are shown in Fig. 5. For the XFM foam for which the fluid and solid phases are strongly coupled, 'resonances' peaks are clearly identifiable. By comparing these results with a simplified bulk-reacting models such as the Limp model, it is concluded that these peaks stem from the elasticity of the frame ${ }^{20}$. In this context, the mounting conditions have a great impact on the dynamic response of the poroelastic liner and its absorbing properties.

The FM4 foam is softer; when considering sliding conditions on the hard wall, the TL curve shows a quite smooth behavior without marked skeleton resonances. Here, results are comparable with the bulkreacting model (Limp model) ${ }^{39}$ and the solid frame influence is essentially due to the inertial coupling. However, when the foam is clamped, the coupling between phases is stronger and the skeleton resonances are clearly visible in the low frequency regime. These effects have been observed especially for small thickness liners.

\section{Shear flow influence}

In this section we shall investigate the effect of a shear flow in the airway on the upstream and downstream propagation for the dissipative silencer of the previous section.

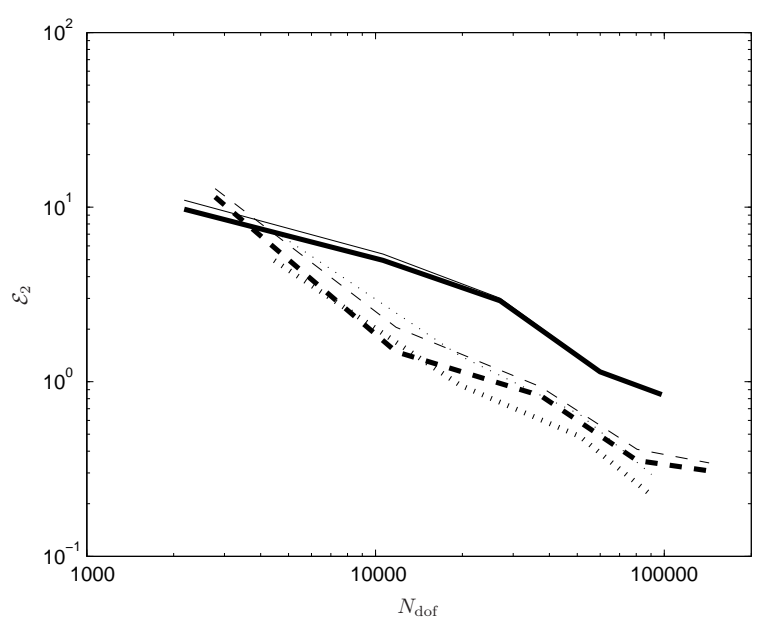

(a)

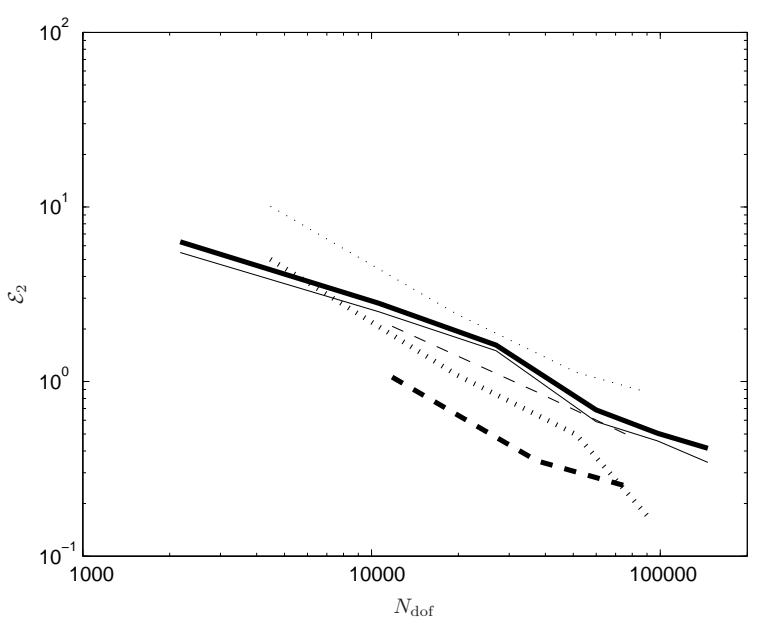

(b)

FIG. 3. Convergence curves for the pressure. The discretization scheme is given by the line style : — LL scheme, $\cdots$ LQ scheme and - - QQ scheme. The line thickness indicates the domain under consideration: bold line for the poroelastic domain and thin line for the fluid domain. (a) No flow case with $\beta=17.135+4.7643$ i. (b) $M=-0.2$ flow with $\beta=20.2572+7.1227$ i.

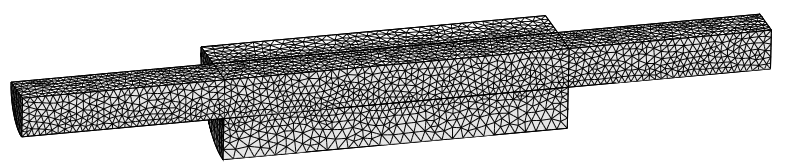

FIG. 4. Test case mesh. 
TABLE III. Materials properties used in numerical tests ${ }^{16,33}$. With the flow resistivity $\sigma$, the tortuosity $\alpha_{\text {inf }}$, the viscous and thermal characteristic lengths $\Lambda$ and $\Lambda^{\prime}$, the poisson coefficient $\nu$ and the effective skeleton density $\rho_{1}$. The effective skeleton density $\rho_{1}=(1-\phi) \rho_{s}$, where $\rho_{s}$ is the density of the material of the frame.

\begin{tabular}{ccccccccc}
\hline \hline Foam & $\phi$ & $\sigma\left[\mathrm{kNm}^{-4} \mathrm{~s}\right]$ & $\alpha_{\text {inf }}$ & $\Lambda[\mu \mathrm{m}]$ & $\Lambda^{\prime}[\mu \mathrm{m}]$ & $\rho_{1}\left[\mathrm{kgm}^{-3}\right]$ & $N[\mathrm{kPa}]$ & $\nu$ \\
\hline XFM & 0.98 & 13.5 & 1.7 & 80 & 160 & 30 & $200(1-0.05 \mathrm{i})$ & 0.35 \\
FM4 & 0.99 & 65 & 1.98 & 37 & 121 & 16 & $18(1-0.1 \mathrm{i})$ & 0.3 \\
\hline \hline
\end{tabular}

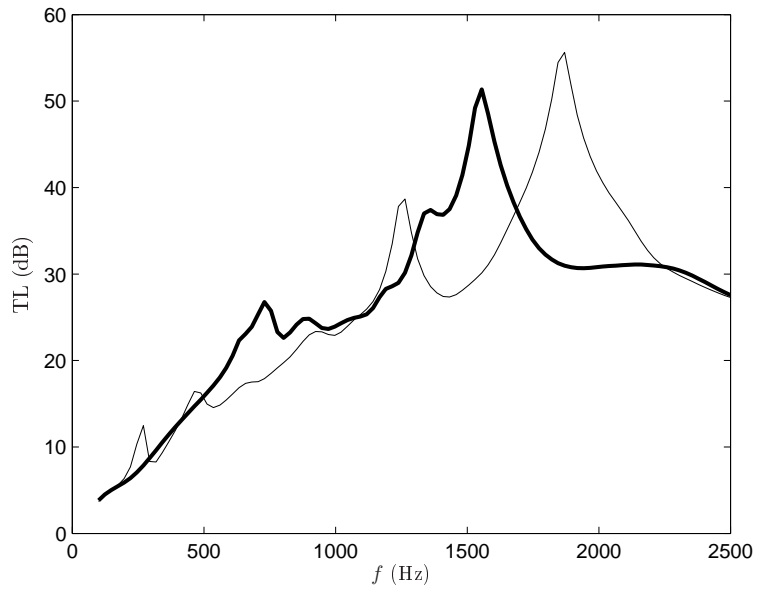

(a)

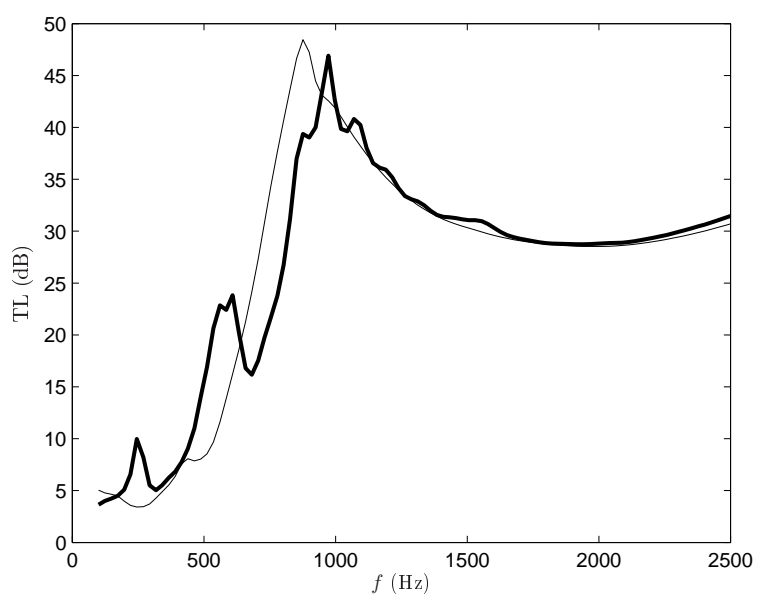

(b)

FIG. 5. Effects of the mounting conditions, - sliding, - clamped, for two foams: (a) XFM, (b) FM4.
The flow profile ${ }^{21}$

$M(r)= \begin{cases}M^{*}, & \text { if } 0 \leq r \leq r_{1}-\delta \\ M^{*} \cos \left(\frac{\pi}{2 \delta}\left(r-\left(r_{1}-\delta\right)\right)\right), & \text { if } r_{1}-\delta<r<r_{1}\end{cases}$

is constant with a magnitude $M^{*}$ excepted in the shear layer, where a sinusoidal transition with a thickness $\delta$ is used. When using a uniform flow model with Mach number $M_{0}$, we made sure that comparisons are made by keeping the same mean Mach number, that is $M_{0}=$ $\overline{M(r)}$. In the following example, the mean Mach number value is chosen equal to 0.2 and the lining material is sliding on the hard walls.

When the flow is uniform, the sound waves propagating upstream are generally more attenuated than when propagating downstream and this is in agreement with a modal analysis (it suffices to compare the imaginary part of the axial wavenumber). Results presented in Fig. 6 for the XFM foam confirm these facts. When the flow is sheared the same conclusion holds, but in addition, the refraction effects in the boundary layer are found to be more significant for the upstream propagation. Note that the convection of the waves by the mean flow changes the 'apparent' wave length which is shorter in the upstream propagation case. This has a noticeable effect on the elasto-acoustic excitation of the lining material. This explains the 'shift' of the resonance peaks on the TL curves.

Refractions effects due to the flow are conveniently illustrated in Fig. 7. Arrows corresponding to the intensity vector field calculated from (12) are shown. This clearly illustrates the noticeable differences between the shear flow and the uniform flow case. For the downstream propagation, the shear flow makes the acoustic intensity penetrate deeper in the poroelastic lining. On the contrary, the shear flow tends to make the intensity of upstream propagating acoustic waves more parallel to the duct axis. These observations are in line with results of Fig. 6, especially the upstream propagation case for which Losses are found to be higher for uniform flows in a large part of the spectrum.

\section{Presence of a perforated screen}

In many problems of practical interest, the presence of a perforated screen separating the absorbing material from the airway is sometimes inevitable. The reasons for this are either to avoid the flow to penetrate through the liner or for other purely practical reasons. The presence of the screen will in turn affect the performance of the dissipative silencer. Because of this, our FE model needs 


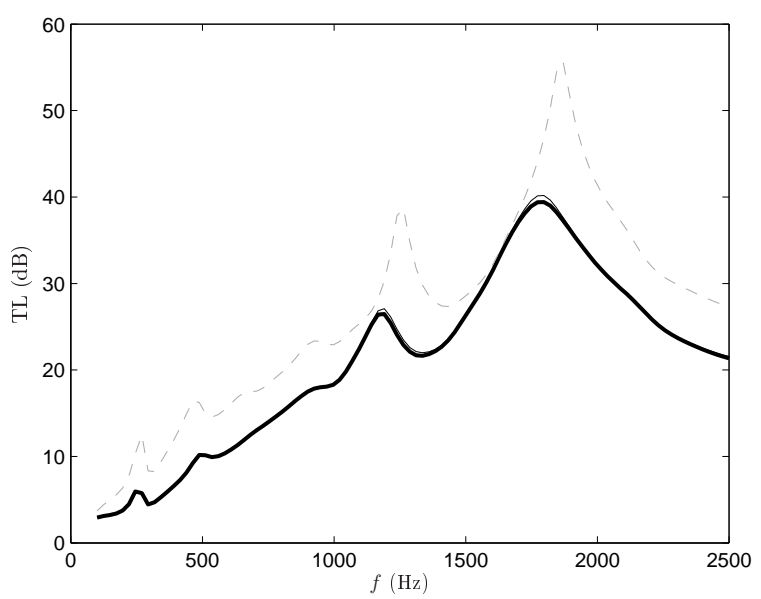

(a)

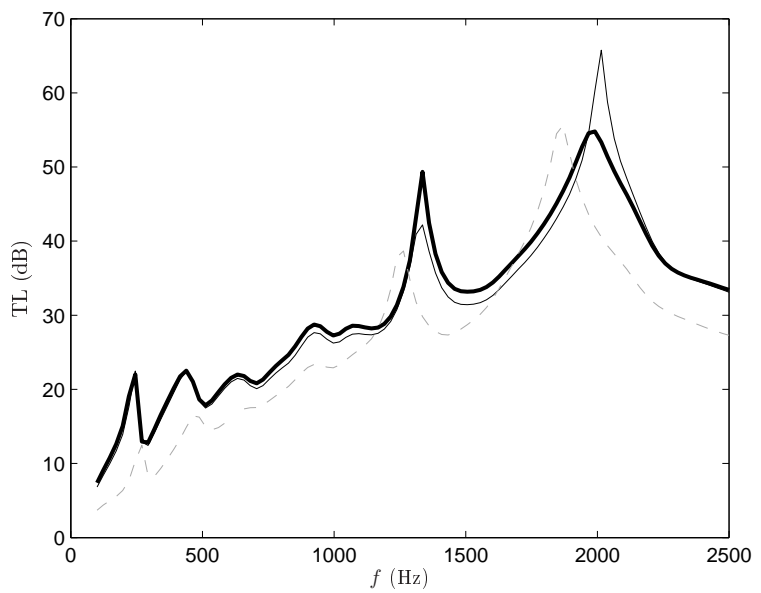

(b)

FIG. 6. Effects of the shear layer with a mean Mach number $M_{0}=0.2,--$ no flow, $-\delta=0.2$, - uniform, for XFM foam. (a) Downstream propagation, (b) Upstream propagation.

to be modified in order to take into account the induced change of pressure $\delta p$ over the perforated screen. Here, the difficulty lies in having appropriate values for the layer impedance defined as the ratio between the change of pressure and the normal velocity at the surface of the liner. These values are usually measured experimentally and various semi-empirical models have been proposed in the literature. A complete survey would be too lengthy to be inserted in this paper and we can refer to recent research works in this field ${ }^{18}$. In particular it was shown that the presence of the grazing flow in the airway and the porous backing behind the screen can have a substantial effect on the impedance ${ }^{42}$.

In the present work we assume in our modeling that the lining material is not directly in contact with the screen so that the normal stress at the surface of the poroelastic liner is due to the air pressure only. The transmission conditions at the interface $\Gamma$ now read

$$
\begin{aligned}
\boldsymbol{\sigma}^{t} \mathbf{n} & =-(p-\mathrm{i} \omega Z \mathbf{w} \cdot \mathbf{n}) \mathbf{n}, \\
\delta p & =p_{p}-p=-\mathrm{i} \omega Z \mathbf{w} \cdot \mathbf{n} .
\end{aligned}
$$

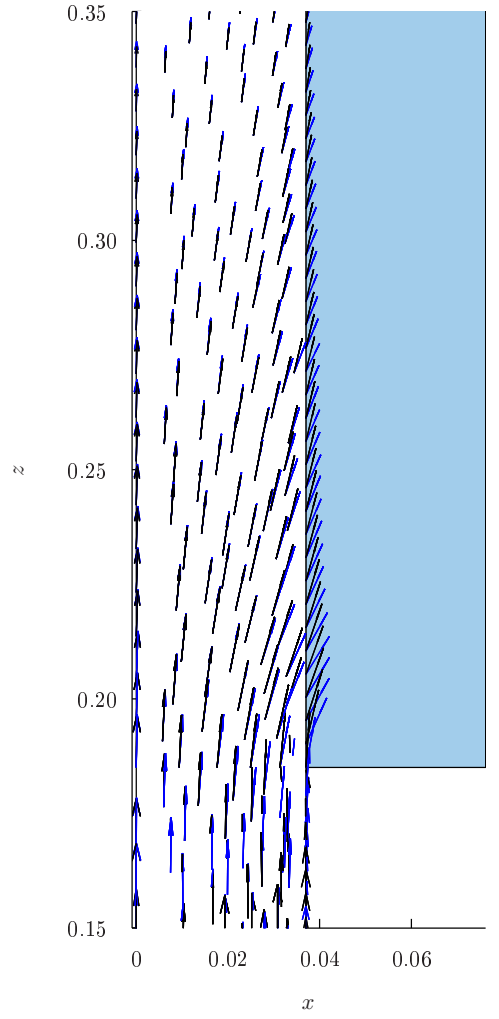

(a)

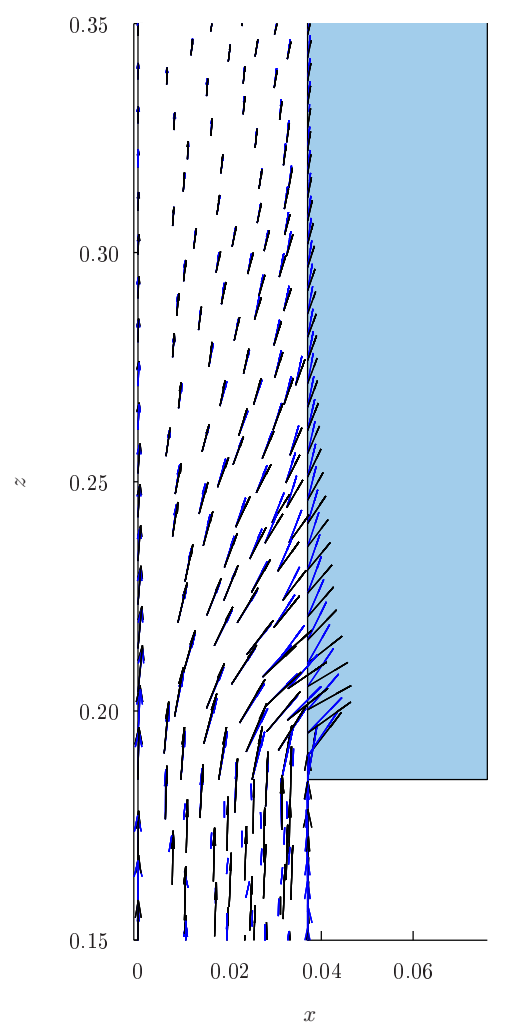

(b)

FIG. 7. Acoustic intensity field at the entrance of the liner at $1021 \mathrm{~Hz},-$ uniform flow; - shear flow. (a) Downstream case, (b) Upstream case. 


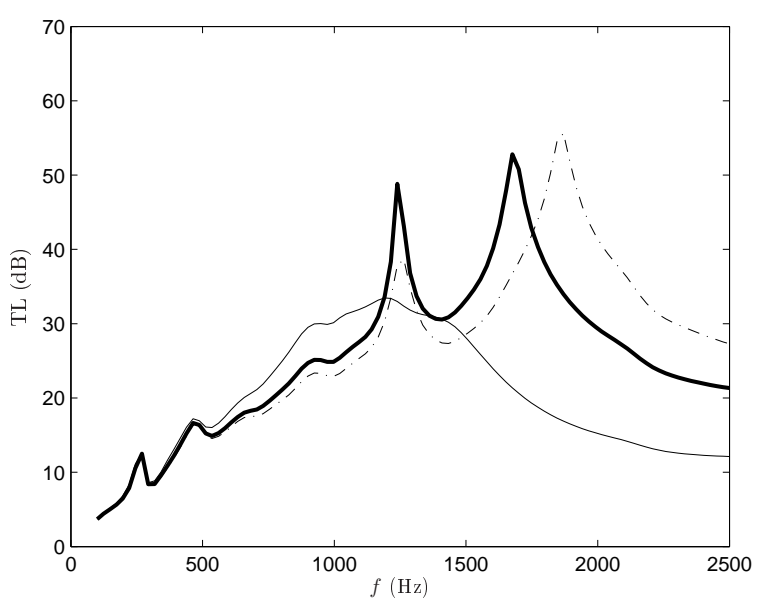

FIG. 8. Effects of the perforated plate properties on the TL (sliding condition for the foam). - plate 2, - plate 1 , - - no plate.

(note that the second equation is usually written in terms of the normal acoustic velocity $v_{n}=-i \omega \mathbf{w} \cdot \mathbf{n}$ on the screen). The last condition stems from the continuity of the normal displacement at each side of the screen and (6c) remains unchanged. Finally, the presence of the perforated screen is taken into account in our FE model by simply adding the boundary integral

$$
-\mathrm{i} \omega \int_{\Gamma} \mathbf{w}^{*} \cdot \mathbf{n} Z \mathbf{w} \cdot \mathbf{n} \mathrm{d} \Gamma
$$

in the Galbrun's matrix G.

In the next example, we shall investigate the noflow case only. For a sake of illustration we chose the impedance model given in Ref. 43

$$
\begin{aligned}
& \frac{Z}{\rho_{0} c_{0}}=\left[\frac{\sqrt{8 v \omega}}{\phi_{p} c_{0}}\left(1+\frac{\tau}{d}\right)+\frac{1}{8 \phi_{p}}\left(k_{0} d\right)^{2}\right] \\
& -\mathrm{i} \frac{\omega}{\phi_{p} c_{0}}\left[\tau+\frac{8}{3 \pi} d\left(1-0.7 \sqrt{\phi_{p}}\right)+\sqrt{\frac{8 v}{\omega}}\left(1+\frac{\tau}{d}\right)\right],
\end{aligned}
$$

with $v=1.510^{-5} \mathrm{~m}^{2} \mathrm{~s}^{-1}$ the air kinematic viscosity, the screen thickness $\tau$, the hole diameter $d$ and the screen porosity $\phi_{p}$. Results are depicted in Fig. 8 for the two screens described in Table IV.

The screen 1 is acoustically transparent below $500 \mathrm{~Hz}$. In the mid frequency range (say $500 \mathrm{~Hz}$ up to $1400 \mathrm{~Hz}$ ) the apparent resistivity increases and a gain of few decibels can be achieved. However, above $1400 \mathrm{~Hz}$, performances drastically decrease because the perforated screen tends to behave like a rigid wall (high impedance value) and the air-porous coupling becomes less effective. For the screen 2, similar trends hold, however the porosity is relatively high and the coupling remains effective in a wider frequency range.
TABLE IV. Perforated screen characteristics.

\begin{tabular}{cccc}
\hline \hline Screen & $d[\mathrm{~mm}]$ & $\tau[\mathrm{mm}]$ & $\phi_{p}[-]$ \\
\hline 1 & 1 & 1 & .05 \\
2 & 3.5 & 1 & .263 \\
\hline \hline
\end{tabular}

\section{E. Notes on computational aspects}

Meshes are built using the free software $\mathrm{Gmsh}^{44}$, the FE implementation (integration and matrix assembly) is carried out on Matlab and the final system is solved with a Fortran-based solver. For homogenous porous material, all frequency dependent parameters of the Biot's model can be pulled out of the integrals. Thus, for a fixed mesh the numerical integration over the elements is performed only once. The system is solved with a direct parallel sparse solver: Mumps (with PORD ordering) ${ }^{45}$ on 8 processors on the High-performance computing platform Pil$\mathrm{cam}^{46}$. To give an idea of the computational cost, the sparse system $\left(\mathrm{nnz}=6,589,999\right.$ and $\left.N_{\text {dof }}=78,574\right)$ corresponding to the mesh of Fig. 4 is solved in 17 seconds and requires 250 Mbytes per working processor.

\section{CONCLUSION}

In this paper, a finite element model for analyzing the sound transmission in ducted shear flows with finite poroelastic lining was presented. The numerical model has been developed for general 3D configurations. The use of a displacement-pressure formalism for both propagative domains (absorbing material and the airway) allows an efficient and natural coupling at the air-porous interface. Comparisons with analytical solutions show the efficiency of the method. It is demonstrated that the numerical model presented in this work gives a complete computational tool for tackling complex configurations of practical interest.

To illustrate the method, the case of a circular dissipative silencer is discussed. In particular, the effects of the mounting conditions of the porous foam on the elasticity of the skeleton are shown. In some cases, elastic resonances are clearly identified and this can have significant consequences on the sound attenuation.

Using the acoustic intensity defined in (12) for arbitrarily mean flow, refraction effects in the boundary layer are shown for the downstream and the upstream propagation. In the case of the fundamental mode, these effects are noticeable only in the upstream case. Work is ongoing by the authors to extend the results when higher order propagative modes are also present in the airway.

Comparisons with experimental data would also be interesting and very instructive, in particular to identify the effect of an internal flow in the absorbent on the porous material properties ${ }^{2}$. This aspect was ignored in the present work but we think that this could be an interesting subject for further investigation. 


\section{Acknowledgement}

The authors acknowledge the Projet Pluri-Formations PILCAM2 at the Université de Technologie de Compiègne for providing HPC resources that have contributed to the research results reported within this paper $^{46}$.

\section{APPENDIX A: MODE CALCULATION FOR AN UNIFORM FLOW}

In this section, the silencer chamber is assumed to be infinite in length and a uniform mean flow is present in the airway. The eigenvalue analysis is performed by assuming that all perturbative quantities have the $\mathrm{e}^{\mathrm{i}(\beta z-\omega t)}$ dependence. Within these assumptions, the displacement perturbation $\mathbf{w}$ satisfies the convected wave equation in the airflow domain (see (1))

$$
\nabla \nabla \cdot \mathbf{w}+\Omega^{2} \mathbf{w}=0
$$

with $\Omega=M \beta-k_{0}, k_{0}=\omega / c_{0}$. Since the flow is uniform, we can look for purely acoustic mode by putting $\mathbf{w}=\nabla \varphi_{0}$ and the acoustic pressure is simply obtained from $p=\rho_{0} c_{0}^{2} \Omega^{2} \varphi_{0}$. In the axisymmetric case, we find that $\varphi_{0}(r)=A_{0} J_{0}\left(\alpha_{0} r\right)$ where the transverse wavenumber satisfies the dispersion relation $\alpha_{0}^{2}+\beta^{2}=\Omega^{2}$. The fluid and solid phase displacements in the porous materials are governed by the Biot's model (3). When the medium is homogeneous, both displacements admit the Helmholtz decomposition $\mathbf{u}=\nabla\left(\varphi_{1}+\varphi_{2}\right)+\nabla \times \boldsymbol{\Psi}$ and $\mathbf{U}=\nabla\left(\mu_{1} \varphi_{1}+\mu_{2} \varphi_{2}\right)+\mu_{3} \nabla \times \boldsymbol{\Psi}$ where potentials $\varphi_{1}$, $\varphi_{2}$ and $\boldsymbol{\Psi}$ are solutions of the Helmholtz equation with wavenumbers $k_{1}, k_{2}$ and $k_{3}$, (see $\left.{ }^{11}\right)$. So we put

$$
\begin{aligned}
& \varphi_{e}(r)=A_{e} J_{0}\left(\alpha_{e} r\right)+B_{e} Y_{0}\left(\alpha_{e} r\right), \quad e=1,2, \\
& \varphi_{3}(r)=A_{3} J_{1}\left(\alpha_{3} r\right)+B_{3} Y_{1}\left(\alpha_{3} r\right),
\end{aligned}
$$

where $\alpha_{e}^{2}+\beta^{2}=k_{e}^{2}(e=1,2,3)$. Note that, for the sake of simplicity, the shear wave potential $\varphi_{3}$ simply stands for the azimuthal component of $\boldsymbol{\Psi}$. The modal vector $\mathbf{V}=\left[A_{2}, B_{2}, A_{3}, B_{3}, A_{1}, B_{1}, A_{0}\right]^{T}$ must be found so that the transmission conditions $(6 \mathrm{a}),(6 \mathrm{~b})$ and $(6 \mathrm{c})$ at the fluid-porous interface $\left(r=r_{1}\right)$ are satisfied. For this, the pore pressure $p_{p}$ and the total stress tensor $\boldsymbol{\sigma}^{t}$ must be expressed in terms of the potentials as shown in ${ }^{11}$. Similarly, at the wall $\left(r=r_{2}\right)$, the foam is clamped, i.e. $\mathbf{u}=0$ and $\mathbf{U} \cdot \mathbf{n}=0$. All these conditions leads to the nonlinear eigenvalue problem

$$
\mathbf{M}(\beta) \mathbf{V}=\mathbf{0}
$$

where $\mathrm{M}(\beta)$ is a $7 \times 7$ matrix. We can now exploit the fact that $\operatorname{det} \mathrm{M}(\beta)$ is a meromorphic function in the complex $\beta$-plane and solve $\operatorname{det} \mathrm{M}(\beta)=0$ using the argument principle. All simple zeros $\beta_{k}^{ \pm}(k=1, \ldots, K)$ are classified with the convention that superscript \pm stands for the sign of the imaginary part (+ refers to rightgoing modes and - to leftgoing modes).
1 R. J. Astley and A. Cummings, "A finite element scheme for attenuation in ducts lined with porous material: comparison with experiment", J. Sound Vib. 116, 239-263 (1987).

2 A. Cummings and I.-J. Chang, "Sound attenuation of a finite length dissipative flow duct silencer with internal mean flow in the absorbent", J. Sound Vib. 127, 1-17 (1987).

${ }^{3}$ W. Eversman and D. Okunbor, "Aft fan duct acoustic radiation", J. Sound Vib. 213, 235-257 (1998).

${ }^{4}$ K. S. Peat and K. L. Rathi, "A finite element analysis of the convected acoustic wave motion in dissipative silencers", J. Sound Vib. 184, 529-545 (1995).

5 A. Craggs, "A finite element model for rigid porous absorbing materials", J. Sound Vib. 61, 101-111 (1978).

${ }^{6}$ L. L. Beranek, "Acoustical properties of homogeneous, isotropic rigid tiles and flexible blankets", J. Acoust. Soc. Am. 19, 556-568 (1947).

7 O. Doutres, N. Dauchez, J.-M. Génevaux, and O. Dazel, "Validity of the limp model for porous materials: a criterion based on the Biot theory", J. Acoust. Soc. Am. 122, 2038-2048 (2007).

8 C. Zwicker and C. W. Kosten, Sound Absorbing Materials (Elsevier, Amsterdam) (1949, 321pp).

${ }^{9}$ M. E. Delany and E. N. Bazley, "Acoustical properties of fibrous absorbent materials", Appl. Acoust. 3, 105-116 (1970).

10 M. A. Biot, "Theory of propagation of elastic waves in a fluid-saturated porous solid. I low-frequency range.", J. Acoust. Soc. Am. 28, 168-191 (1956).

11 J.-F. Allard, Propagation of Sound in Porous Media: Modeling Sound Absorbing Materials (Chapman \& Hall) (1993, 280pp).

${ }^{12}$ Y. J. Kang and J. S. Bolton, "Finite element modeling of isotropic elastic porous materials coupled with acoustical finite elements", J. Acoust. Soc. Am. 98, 1635-643 (1995).

13 N. Atalla, M. A. Hamdi, and R. Panneton, "Enhanced weak integral formulation for the mixed $(u, p)$ poroelastic equations", J. Acoust. Soc. Am. 109, 3065-3068 (2001).

14 N. E. Horlin, M. Nordstron, and P. Goransson, "A 3-D hierarchical FE formulation of Biot's equations for elastoacoustic modeling of porous media", J. Sound Vib. 245, 633-652 (2001).

15 S. Rigobert, N. Atalla, and F. Sgard, "Investigation of the convergence of the mixed displacement-pressure formulation for three-dimensional poroelastic materials using hierarchical elements", J. Acoust. Soc. Am. 114, 2607-2617 (2003).

16 N. Atalla, R. Panneton, and P. Debergue, "A mixed displacement-pressure formulation for poroelastic materials", J. Acoust. Soc. Am. 104, 1444-1452 (1998).

17 Y. J. Kang and I. H. Jung, "Sound propagation in circular ducts lined with noise control foams", J. Sound Vib. 239, 255-273 (2001).

18 R. Kirby, "Simplified techniques for predicting the transmission loss of a circular dissipative silencer", J. Sound Vib. 243, 403-426 (2001).

19 S. W. Rienstra, "Contributions to the theory of sound propagation in ducts with bulk-reacting lining", J. Acoust. Soc. Am. 77, 1681-1685 (1985).

20 B. Nennig, E. Perrey-Debain, and M. Ben Tahar, "A mode matching method for modelling dissipative silencers lined with poroelastic materials and containing mean flow", J. Acoust. Soc. Am. 128, 3308-3320 (2010).

21 W. Eversman, "Effect of Boundary Layer on the Transmission and Attenuation of Sound in an Acoustically Treated Circular Duct", J. Acoust. Soc. Am. 49, 1372-1380 (1971). 
${ }^{22}$ G. R. Gogate and M. L. Munjal, "Sound propagation in ducts with bulk reacting lining in the presence of laminar mean flow", J. Acoust. Soc. Am. 99, 1779-1782 (1996).

23 D. C. Pridmore-Brown, "Sound propagation in a fluid flowing through an attenuating duct", J. Fluid Mech. 4, 393406 (1958).

${ }^{24}$ H. Galbrun, Propagation d'une onde sonore dans l'atmosphère terrestre et théorie des zones de silence (Propagation of an acoustic wave in the atmosphere and theory of zones of silence) (Gauthier-Villars, Paris) (1931, 352pp).

25 O. A. Godin, "Reciprocity and energy theorems for waves in a compressible inhomogeneous moving fluid", Wave motion 25, 143-167 (1997).

${ }^{26}$ R. J. Astley, "Numerical methods for noise propagation in moving flows, with application to turbofan engines", Acoust. Sci. Tech. 30, 227-239 (2009).

27 B. Poirée, "Les équations de l'acoustique linéaire et non linéaire dans un écoulement de fluide parfait (equations of linear and non linear acoustics in a perfect fluid flow)", Acustica 57, 5-25 (1985).

${ }^{28}$ F. Treyssède, G. Gabard, and M. Ben Tahar, "A mixed finite element method for acoustic wave propagation in moving fluids based on an eulerian lagrangian description", J. Acoust. Soc. Am. 113, 705-716 (2003).

29 G. Gabard, F. Treyssède, and M. B. Tahar, "A numerical method for vibro-acoustic problems with sheared mean flows", J. Sound Vib. 272, 991-1011 (2004).

30 M. E. Goldstein, Aeroacoustics (McGraw-Hill, New York) (1976, 293pp).

${ }^{31}$ F. Treyssède and M. Ben Tahar, "Comparison of a finite element model with a multiple-scales solution for sound propagation in varying ducts with swirling flows", J. Acoust. Soc. Am. 115, 2716-2730 (2004).

32 A. S. Bonnet-BenDhia, E. M. Duclairoir, G. Legendre, and J.-F. Mercier, "Time-harmonic acoustic propagation in the presence of a shear flow", J. Comput. Appl. Math 204, 428-439 (2007).

${ }^{33}$ P. Debergue, R. Panneton, and N. Atalla, "Boundary conditions for the weak formulation of the mixed $(u, p)$ poroelasticity problem", J. Acoust. Soc. Am. 106, 2393-2390 (1999).

${ }^{34}$ G. Gabard, R. J. Astley, and M. Ben Tahar, "Stability and accuracy of finite element methods for flow acoustics. II: Two-dimensional effects", Int. J. Num. Meth. Eng. 63, 947-973 (2005).

35 M. K. Myers, "On the acoustic boundary condition in the presence of flow", J. Sound Vib. 71, 429-434 (1980).

${ }^{36}$ K. Bathe, Finite element procedures (Prentice Hall, Englewood Cliffs) (1996, 1040pp).

37 H. Bériot and M. Ben Tahar, "A three dimensional finite element model for sound propagation in non potential mean flows", in $13^{\text {th }} \operatorname{ICSV}$ (Vienna, Austria) (July 2-6, 2006).

${ }^{38}$ P. Mungur and G. M. L. Gladwell, "Acoustic wave propagation in a sheared fluid contained in a duct", J. Sound Vib. 28-48 (1969).

39 B. Nennig, "Contribution à l'étude de matériaux absorbants acoustiques en présence d'écoulement (a contribution to acoustic absorbing materials understanding in the presence of a mean flow)", Ph.D. thesis, Université de Technologie de Compiègne, France (2010) (120pp).

40 C. Peyret and G. Élias, "Finite-element method to study harmonic aeroacoustics problems", J. Acoust. Soc. Am. 110, 661-668 (2001).

41 D. Pilon and R. Panneton, "Behavioral criterion quantifying the edge-constrained effects on foams in the standing wave tube", J. Acoust. Soc. Am. 114, 1980-1987 (2003).
${ }^{42}$ R. Kirby and A. Cummings, "The impedance of perforated plates subjects to grazing gas flow and backed by porous media", J. Sound Vib. 217, 619-636 (1998).

43 A. W. Guess, "Calculation of perforated plate liner parameters from specified acoustic resistance and reactance", J. Sound Vib. 40, 119-137 (1975).

44 C. Geuzaine and J.-F. Remacle, "Gmsh: a threedimensional finite element mesh generator with built-in pre- and post-processing facilities", Int. J. Num. Meth. Eng. 79, 1309-1331 (2009).

45 P. R. Amestoy, I. S. Duff, and J.-Y. L'Excellent, "Multifrontal parallel distributed symmetric and unsymmetric solvers", Comput. Methods in Appl. Mech. Eng. 184, 501$520(2000)$.

46 http://pilcam2.wikispaces.com (date last viewed 10/26/2010). 